\title{
Infraestructura nacional de datos espaciales de Honduras - primeros pasos hacia una integración nacional
}

\author{
Gustavo Adolfo M. Salgado y Nancy Karolina Martínez
}

\section{Resumen:}

La Infraestructura de Datos Espaciales de Honduras, INDES, es un conjunto de políticas, leyes, acuerdos y tecnologías que tiene como fin compartir información geográfica, generando un ambiente de transparencia y seguridad jurídica, por medio de la difusión de la información de manera pertinente y de carácter oficial, buscando la reducción de costos organizacionales a través de la colaboración y el intercambio de información, la captura de datos y su reutilización, creando a partir de esto valor agregado a productos y servicios a través de cadenas de servicios y apoyo en la toma de decisiones.

La INDES está formada y regulada por la Comisión de Datos Espaciales, CIDES, cuya finalidad es identificar, analizar, hacer propuestas, revisar estándares, normativas, procedimientos, apoyar en la definición de competencias institucionales en la temática de cartografía, así como también en todo lo referente a la INDES y demás asuntos que le competen en su área de acción enmarcados en La Ley de OT y su Reglamento.

Para lograr impulsar el desarrollo de la INDES se ha iniciado un largo proceso integral que requiere de voluntad política, generación de capacidades, un gran voluntariado institucional y un presupuesto que la viabilizará, convirtiéndola en un éxito del Gobierno de la República de Honduras.

Como etapa inicial, la SEPLAN por medio del Organismo Hondureño de Normalización (OHN) y CIDES trabajaron en la traducción de las normas ISO relacionadas con los metadatos $(19104,19110,19115,19115-2,19139)$ para la elaboración de la Normativa Hondureña de Metadatos (NOHME) la cual se encuentra en la etapa de implementación y que permitirá describir totalmente los datos geográficos de manera que los usuarios puedan entender los fenómenos descritos o abstraídos de la realidad y limitaciones, y puedan evaluar la aplicabilidad de los datos para el uso específico de su interés. 
Palabras clave: INDES / CIDES / SEPLAN / Normativa / Metadatos

\begin{abstract}
:
The National Spatial Data Infrastructure of Honduras, NSDI, is a set of policies, laws, agreements and technologies that aims to share geographic information, generating an environment of transparency and legal certainty, through the dissemination of information in a relevant and official way, seeking to reduce organizational costs through collaboration and exchange of information, data capture and its reusability, creating from this an added value to products and services through service chains and support for the decision makers.

The NSDI is formed and regulated by the Commission on Spatial Data, CIDES, whose purpose is to identify, analyze, make suggestions, review standards, regulations, procedures, support the definition of institutional competence in the thematic mapping, as well as all regarding the NSDI and other issues that concern in its area of action, framed in the Law of Land Management and its Regulation.

In order to boost the development of the NSDI we have initiated an integrated long process that requires political will, create capacities, a great institutional volunteering and a budget that will make it viable, making it a big success of the Government of the Republic of Honduras.

As an initial step, the SEPLAN through the Honduran Organization for Standardization (OHN) and CIDES have worked in the translation of ISO standards related to metadata $(19104,19110,19115,19115-2,19139)$ for the development of the Honduran Normative of Metadata (NOHME) which is in the phase of implementation and that will fully describe geographic data so that users can understand the phenomena described or abstracted from reality and limitations, and can evaluate the applicability of the data for the specific use of interest.
\end{abstract}

Keywords: NSDI / CIDES / SEPLAN / Normative / Metadata

Gustavo Adolfo M. Salgado (gsalgado@seplan.gob.hn); Nancy Karolina Martínez (nmartinez@seplan.gob.hn). Dirección General de Ordenamiento Territorial - DGOT. Secretaría Técnica de Planificación y Cooperación Externa - SEPLAN. 


\section{INFRAESTRUCTURA NACIONAL DE DATOS ESPACIALES DE HONDURAS}

En Octubre del año 2003 se promulgo la Ley de Ordenamiento Territorial, creada mediante decreto 180-2003, publicada en el Diario Oficial La Gaceta el día martes 30 de diciembre de 2003, en cuyo marco se creó la Dirección General de Ordenamiento Territorial (DGOT) con atribuciones como: establecer la organización y gestión del Sistema Nacional de Información Territorial (SINIT), que permita el manejo de información sobre todos los aspectos relativos al Ordenamiento Territorial; la integración y coordinación de iniciativas provenientes de los centros técnicos citados en el Artículo 51 de la Ley; también será responsable del mantenimiento de la infraestructura del SINIT (entre otras).

El Articulo 47 de la Ley establece como uno de los instrumentos de registro técnicos del Ordenamiento Territorial al Registro Nacional de Normativas del Ordenamiento Territorial (RENOT), el cual según el Artículo 57 del Reglamento "como el sistema especializado registra toda la información territorial que envían las instituciones mencionadas en el Artículo 51 de la Ley, tales como leyes, reglamentos, normativas, ordenanzas, documentos legales, planos y disposiciones que determinen cualquier incidencia de ordenamiento territorial que manejen y que de acuerdo con la Ley regulen o limiten los derechos de uso o disposición de los bienes inmuebles nacionales, municipales y privados."

Así mismo el Artículo 64 del Reglamento establece que "Todas las instituciones que generan, elaboran o utilizan información vinculada y aplicable al Ordenamiento Territorial, están obligadas a incorporar los resultados de sus investigaciones y estudios al SINIT, utilizando para ello los medios y mecanismos consignados en el Reglamento General de la "Infraestructura Nacional de Datos Espaciales (INDES)".

La INDES se compone de reservorios temáticos entre los cuales inicialmente se encuentran: el SINIT y el RENOT. Con la implementación de la INDES se estará regulando la utilización de información espacial, como una herramienta de apoyo técnico en la toma de decisiones para los distintos procesos de planificación territorial que se llevan en el país, en términos generales como una red de socios trabajando desde su propio ámbito y competencia sobre la plataforma tecnológica de Internet, para que los usuarios puedan buscar, ver y utilizar la información espacial generada por el Estado de Honduras. Esta red permite, tanto a los generadores de la información geográfica como a los usuarios (técnicos y 
ciudadanos), el acceso, disponibilidad, uso, reutilización y el intercambio de datos espaciales, así como el desarrollo de aplicaciones específicas para la satisfacción de necesidades puntuales de la sociedad, volviendo de esta forma a la información geográfica de uso y beneficio común. En el País, existe información dispersa (en algunos casos duplicada) en diferentes instituciones, lo que dificulta el acceso a la misma; de ahí la necesidad de la construcción de la INDES.

\section{COMPONENTES DE LA INDES}

La INDES aglutina políticas, redes de acceso y estándares, siendo estos los componentes tecnológicos que permiten la interacción entre las comunidades y los datos fundamentales facilitando la información para investigación, análisis, toma de decisiones, etc. En la Figura 1 se aprecia la dinámica de los componentes y sus elementos propios.

Figura 1 - Componentes de la INDES

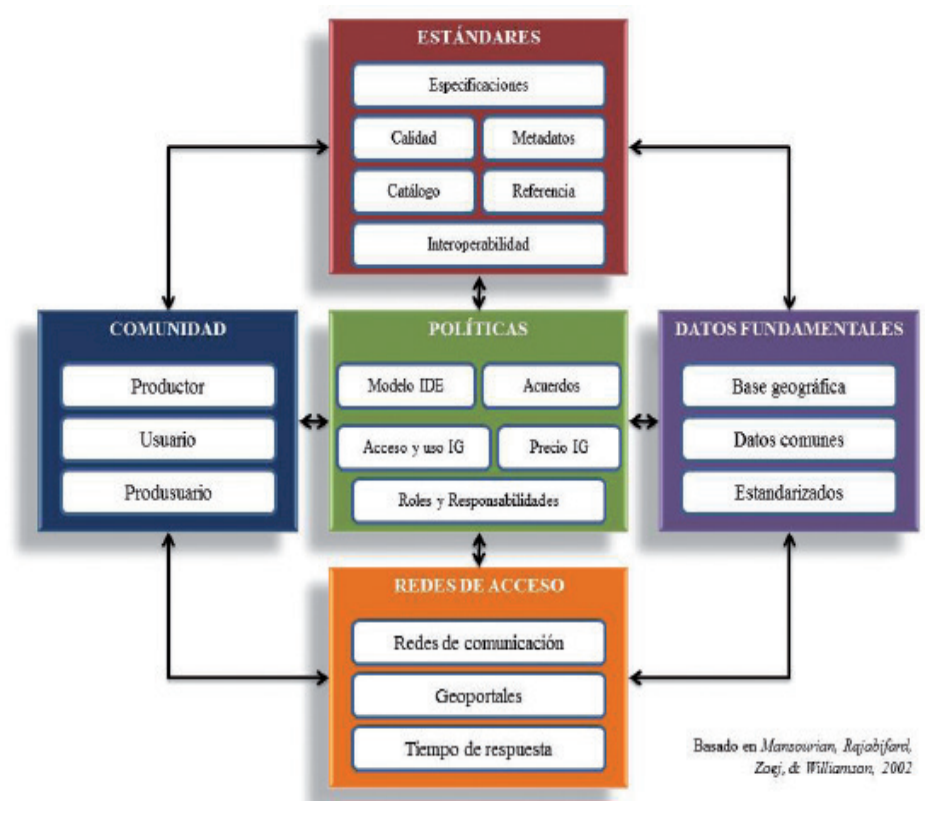

A continuación se presentaran los avances hasta la fecha en cada uno de los componentes para el establecimiento y la puesta en marcha de la INDES de Honduras: 


\subsection{Políticas}

La INDES de Honduras está formada y regulada por la Comisión Interagencial de Datos Espaciales (CIDES) cuya finalidad es identificar, analizar, hacer propuestas, revisar estándares, normativas, procedimientos, apoyar en la definición de competencias institucionales en la temática de cartografía, así como también en todo lo referente a la INDES y demás asuntos que le competen en su área de acción enmarcados en la Ley de Ordenamiento Territorial y su Reglamento, pudiendo referir otros asuntos que considere prioritarios para ser tratados por otras Comisiones que forman parte de la institucionalidad para el ordenamiento territorial.

CIDES está integrada por las siguientes Instituciones o entidades:

- Secretaría Técnica de Planificación y Cooperación Externa (preside), a través de la DGOT

- Instituto de la Propiedad - IP

- Secretaría del Interior y Población - SEIP

- Secretaría de Recursos Naturales y Ambiente - SERNA

- Instituto Nacional de Conservación y Desarrollo Forestal, Áreas Protegidas y Vidas Silvestre - ICF

- Comisión Permanente de Contingencias - COPECO

- Banco Central de Honduras - BCH

- Secretaría de Obras Públicas, Transporte y Vivienda - SOPTRAVI

- El Consejo de Educación Superior - CES

- Consejo Hondureño de la Empresa Privada - COHEP

- Instituto Nacional de Estadísticas - INE

- Secretaría de Estado en el Despacho de la Presidencia - SDP

En calidad de invitados especiales están:

- Instituto Hondureño de Turismo - ITH

- Secretaría de Agricultura y Ganadería - SAG

- Servicio Autónomo Nacional de Acueductos y Alcantarillados - SANAA

- Empresa Nacional de Energía Eléctrica - ENEE

- Secretaría de Educación 
Entre los principales acuerdos logrados para impulsar a la INDES están los siguientes:

- SERNA/PNUD - Fondo de Adaptación al Cambio Climático, apoyando con el Marco Tecnológico de SINIT y RENOT.

- Instituto de la Propiedad - Programa de Administración de la Tierra, apoyando con el desarrollo de la Normativa Hondureña de Metadatos.

- Instituto de Conservación Forestal - Proyecto Modernización del Sector Forestal de Honduras, apoyado con la gestión de la calidad de la información.

\subsection{Datos fundamentales}

La base geográfica es provista a través del SINIT el cual por el Articulo 62 del Reglamento "SINIT consignará información organizada en cuatro grandes áreas:

1. Cartografía básica;

2. Fisiografía y recursos naturales;

3. Infraestructura, equipamiento social; y,

4. Aspectos sociales y económicos."

Dicha información es provista por parte de las instituciones que conforman CIDES y que es puesta a disposición del público en general de forma gratuita por medio de su plataforma web. Dicha información esta estandarizada y codificada aunque cabe señalar que hay información pendiente de actualización, elaborada con distintas metodologías y en diferentes escalas lo que muchas veces imposibilita un análisis geográfico integral.

\subsection{Estándares}

El Artículo 62 de la Ley de Ordenamiento Territorial establece que "La estructura básica del SINIT, consignara información organizada en cuatro grandes áreas: 1) Cartografía básica; 2) Fisiografía y Recursos Naturales; 3) Infraestructura 
y Equipamiento Social; y 4) Aspectos Sociales y Económicos. La información del SINIT, una vez consignada en las anteriores áreas temáticas, será denominada como un "conjunto de datos" (dataset por su nombre en inglés) el cual contará con una ficha técnica en donde se señalaran las fuentes y los derechos de autor, las responsabilidades del contenido, la metodología de elaboración, fechas entre otros datos de base. Esta ficha técnica recibirá el nombre de Metadato."

Bajo esta premisa, y como etapa inicial, la SEPLAN por medio del Organismo Hondureño de Normalización (OHN) y CIDES trabajaron en la traducción de las normas ISO relacionadas con los metadatos (19104, 19110, 19115, 19115-2, 19139) para la elaboración de la Normativa Hondureña de Metadatos (NOHME) la cual se encuentra en la etapa de implementación y que permitirá describir totalmente los datos geográficos de manera que los usuarios puedan entender los fenómenos descritos o abstraídos de la realidad y limitaciones, y puedan evaluar la aplicabilidad de los datos para el uso específico de su interés.

En el documento de la NOHME se establece el Perfil de Metadatos, resumido en la Tabla 1, el cual contiene un conjunto mínimo de metadatos núcleo que permitirá describir los datos geográficos de manera que los productores puedan documentar su información y que los usuarios puedan entender y saber el origen, alcance y calidad de los mismos. 
Tabla Nº1 - Perfil de Metadatos

\begin{tabular}{|c|c|c|c|}
\hline No. & Nombre & $\begin{array}{l}\text { Nombre } \\
\text { (español) }\end{array}$ & Condición \\
\hline & MD_Metadata & Metadatos & - \\
\hline 01 & FileIdentifier & Identificador del fichero & $\mathrm{OP}$ \\
\hline 02 & Language & Idioma & $\mathrm{C}$ \\
\hline 03 & HierarchyLevel & Nivel jerárquico & $\mathrm{C}$ \\
\hline 04 & HierarchyLevelName & Nombre del nivel jerárquico & C \\
\hline 05 & Contact & Contacto & $\mathrm{O}$ \\
\hline 06 & Date Stamp & Fecha de creación & $\mathrm{O}$ \\
\hline 07 & MetadataStandardName & Norma de metadatos & $\mathrm{OP}$ \\
\hline 08 & MetadataStandardVersion & Versión de la norma de metadatos & OP \\
\hline 09 & spatialRepresentationInfo & Información sobre la representación espacial & OP \\
\hline \multirow[t]{2}{*}{10} & IdentificationInfo & Información de identificación & $\mathrm{O}$ \\
\hline & MD Identification & Información de identificación & - \\
\hline 11 & Citation & Mención & $\mathrm{O}$ \\
\hline 12 & Abstract & Resumen & $\mathrm{O}$ \\
\hline 13 & Purpose & Propósito & OP \\
\hline 14 & resourceMaintenance & Mantenimiento del recurso & OP \\
\hline \multirow[t]{2}{*}{15} & Resource Format & Formato del recurso & OP \\
\hline & MD_DataIdentification & Identificación de datos & - \\
\hline 16 & Spatial Representation Type & Tipo de representación espacial & OP \\
\hline 17 & Language & Idioma & $\mathrm{O}$ \\
\hline 18 & Environment Description & Descripción del entorno & OP \\
\hline
\end{tabular}




\begin{tabular}{|c|c|c|c|}
\hline No. & Nombre & $\begin{array}{l}\text { Nombre } \\
\text { (español) }\end{array}$ & Condición \\
\hline \multirow[t]{2}{*}{19} & Extent & Extensión & $\mathrm{OP}$ \\
\hline & MI_Keywords & Palabras clave & - \\
\hline \multirow[t]{2}{*}{20} & Keyword & Palabras clave & $\mathrm{O}$ \\
\hline & MD_Resolution & Resolución & - \\
\hline \multirow[t]{2}{*}{21} & EquivalentScale & Escala equivalente & $\mathrm{C}$ \\
\hline & MD_Constraints & Información de restricciones & - \\
\hline \multirow[t]{2}{*}{22} & useLimitation & Limitación de uso & $\mathrm{OP}$ \\
\hline & DQ_DataQuality & Calidad de datos & $\mathrm{O}$ \\
\hline 23 & Scope & Ámbito & $\mathrm{O}$ \\
\hline 24 & Report & Informe & $\mathrm{C}$ \\
\hline \multirow[t]{2}{*}{25} & Lineage & Linaje & $\mathrm{C}$ \\
\hline & DQ_Element & Elemento de calidad & $\mathrm{O}$ \\
\hline \multirow[t]{2}{*}{26} & cvaluationMcthodType & Tipo de método de cvaluación & $\mathrm{OP}$ \\
\hline & MD_Reference System & Sistema de Referencia & O \\
\hline \multirow[t]{2}{*}{27} & Reference system identifier & Identificador del sistema de referencia & C \\
\hline & MD_CRS & MD_Sistema de coordenadas & - \\
\hline 28 & Projection & Proyección & $\mathrm{OP}$ \\
\hline 29 & Ellipsoid & Elipsoide & OP \\
\hline 30 & Datum & Datum & OP \\
\hline 31 & Ellipsoid parameters & Parámetros del elipsoide & OP \\
\hline \multirow[t]{2}{*}{32} & Projection parameters & Parámetros de la proyección & OP \\
\hline & MD_Distribution & Distribución & $\mathrm{O} / \mathrm{C}$ \\
\hline 33 & Distribution format & Formato de distribución & C \\
\hline \multirow[t]{2}{*}{34} & Distributor & Distribuidor & OP \\
\hline & CI Contact & Cl Contacto & 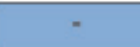 \\
\hline 35 & Address & Dirección & OP \\
\hline 36 & onLineResource & Recurso en línea & OP \\
\hline
\end{tabular}




\subsection{Redes de acceso}

Las redes de acceso son un punto toral dentro de la construcción de la INDES pues en ella se refleja los avances de los componentes anteriores, es la parte tecnológica que enlaza los esfuerzos de las políticas, acuerdos interinstitucionales, la implementación de los estándares, la calidad de los datos geográficos, etc. con el fin de ponerlos a la disposición de los usuarios.

Bajo esta premisa la DGOT ha realizado un trabajo de investigación y autoaprendizaje de tecnologías open source que permitan el desarrollo de geopórtales y servicios en línea estables, a un bajo costo de desarrollo y de mantenimiento, y que sobre todo se pueda replicar en otras instituciones del Estado para poder afianzar una comunidad proactiva a nivel de Gobierno.

A partir de este esfuerzo en término de un año y medio se han logrado crear el geopórtal de SINIT, conteniendo Cartografía Básica; Fisiografía y Recursos Naturales; Infraestructura, Equipamiento Social; y, Aspectos Sociales y Económicos; y el geopórtal de RENOT, conteniendo información relacionada con restricciones de uso del suelo y Planes de Ordenamiento Territorial en los distintos niveles territoriales.

Figura N² 2 - Geopórtal SINIT (http://www.sinit.hn/)

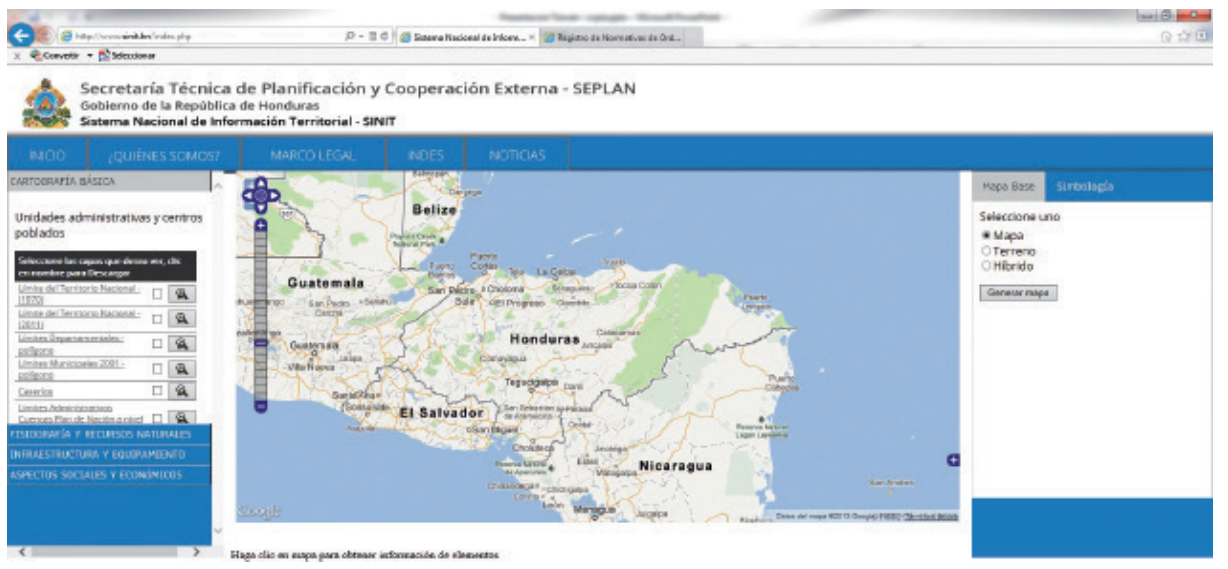


Figura N 3 - Geopórtal RENOT (http://www.renot.hn/)

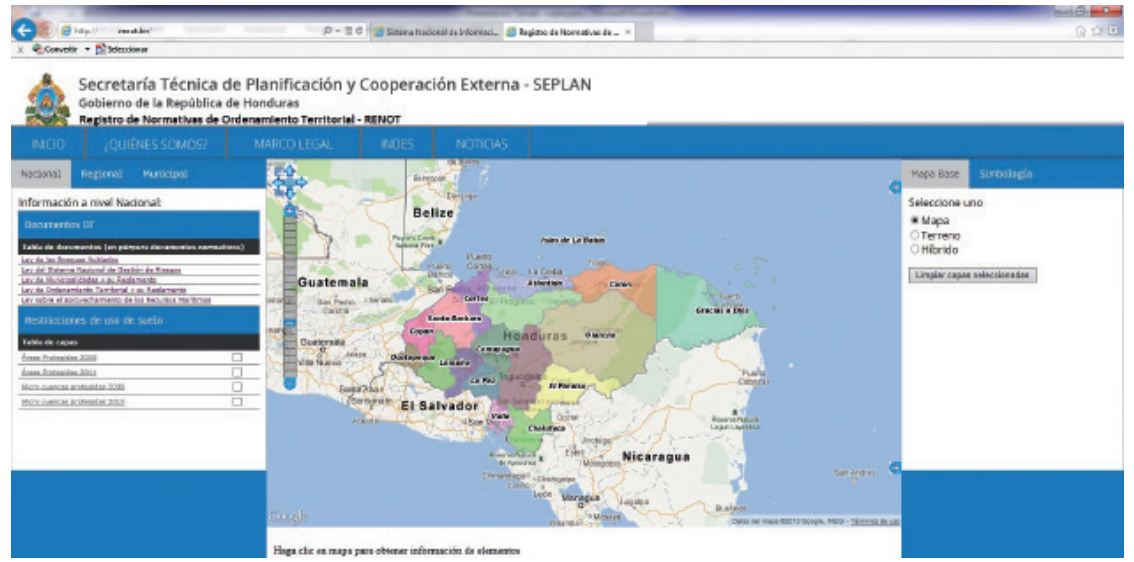

Entre las tecnologías open source utilizadas para ambas plataformas están:

- Postgre SQL

- PostGIS Database

- Geoserver

- OpenLayers

- Javascript

\subsection{Comunidad}

Actualmente se está consolidando poco a poco una comunidad se usuarios y productores interinstitucionales por medio de CIDES, en la medida en que cada institución elabore su geopórtal, poniendo a disposición los servicios web así como su catálogo de metadatos, la INDES ira creciendo y afianzándose más y más cada día, entre los principales miembros activos tenemos:

- Secretaría Técnica de Planificación y Cooperación Externa - SEPLAN

- Secretaría de Recursos Naturales y Ambiente - SERNA

- Secretaría en el Despacho de Relaciones Exteriores - SDRE 
- Secretaria del Interior y Población - SEIP

- Secretaría de Agricultura y Ganadería - SAG

- Instituto de la Propiedad - IP

- Programa de Administración de Tierras de Honduras - PATH

- Instituto Nacional de Conservación y Desarrollo Forestal, Áreas Protegidas y Vidas Silvestre - ICF

- Instituto Hondureño de Turismo - ITH

- Comisión Permanente de Contingencias - COPECO

- Consejo de Educación Superior - CES

- Consejo Hondureño de la Empresa Privada - COHEP

- Servicio Autónomo Nacional de Acueductos y Alcantarillados - SANAA

- Empresa Nacional de Energía Eléctrica - ENEE

- Centro Regional de Documentación e Interpretación Ambiental - CREDIA

\section{RESULTADOS / OPORTUNIDADES}

\section{Resultados}

- Implementado un proceso de armonización e iniciado un proceso de reglamentación para la producción de datos geográficos.

- Implementadas tecnologías estandarizas para la interoperabilidad de las plataformas SINIT y RENOT con otras en el marco de la INDES.

- Definición de normativas, procesos para la gestión de las bases de datos y su publicación.

- Puesto a disposición del público en general la información espacial (103 capas) y normativas (600 documentos) generada por el gobierno de Honduras.

\section{Oportunidades}

- Vinculación de datos espaciales como insumo al gobierno electrónico.

- Reglamento Regulador de la Infraestructura de Datos Espaciales, aprobada por la CIDES.

- Plan cartográfico para la creación de servicios estatales a partir de la cartografía, 
que permitan hacer auto sostenible el proceso y contribuyan al uso masivo de la información y al desarrollo del gobierno electrónico, generando un ambiente de trasparencia y seguridad jurídica en el País. Asignación posible la Universidad Autónoma de Honduras por medio de la Facultad de Ciencias Espaciales.

- Aplicación de estándares de para la producción, manejo y publicación de la información geográfica.

- Gestión del conocimiento formal y capacitaciones puntuales, para fortalecer las capacidades y procesos en el manejo de los datos espaciales.

- Alineamiento de los procesos de producción y gestión de la información.

- Mapeo de la gestión de los recursos para su seguimiento y evaluación en los procesos de planificación.

- Creación de geopórtales temáticos estandarizados sobre el territorio.

- Desarrollo de capacidades en el uso de la información geográfica para en los procesos de planificación.

\section{BIBLIOGRAFÍA}

- Honduras. Ley de Ordenamiento Territorial, Decreto No. 180-2003, del 30 de octubre de 2003. La Gaceta, 30 de diciembre de 2003, No. 30277.

- Honduras. Reglamento General de la Ley de Ordenamiento Territorial, Acuerdo No. 25-2004, del 02 de agosto de 2004. La Gaceta, 18 de septiembre de 2004, No. 30497 .

- Honduras. Secretaría Técnica de Planificación y Cooperación Externa. (2012). Normativa Hondureña de Metadatos - NOHME. Tegucigalpa: DGOT.

- Rajabifard, A., Mansourian, A., Valadan Zoej, M. J. and Williamson I. P. (2004). "Developing Spatial Data Infrastructure to Facilitate Disaster Management", Proceedings of Geomatics 83, National Cartographic Center (NCC), Tehran, Iran. 